\title{
ATHWART IMMERSIONS IN EUCLIDEAN SPACE
}

\section{S. A. ROBERTSON and F. J. CRAVEIRO de CARVALHO}

(Received 21 October 1982, revised 28 March 1983)

Communicated by K. Mackenzie

\begin{abstract}
Let $f$ and $g$ denote immersions of the $n$-manifolds $M$ and $N$, respectively, in $R^{n+1}$. We say that $f$ is athwart to $g$ if $f(M)$ and $g(N) \mathrm{m}$ have no tangent hyperplane in common. In this paper necessary conditions for athwartness are obtained.
\end{abstract}

1980 Mathematics subject classification (Amer. Math. Soc.): 57 R 42, 53 A 04, 53 A 05, 53 A 07.

\section{Introduction}

We study the following problem. Let $M$ and $N$ be smooth $\left(=C^{\infty}\right)$, closed, connected manifolds and let $f$ and $g$ be smooth immersions of $M$ and $N$, respectively, in Euclidean $(n+1)$-space $R^{n+1}$. We say that $f$ is athwart to $g$, written $f A g$, if and only if $f(M)$ and $g(N)$ have no tangent hyperplane in common. In what circumstances is $f A g$ ?

It is easy to think of instances where $f A g$ and others where $f$ is not athwart to g. Some examples, for the case $n=1$, are shown in Figure 1 below. In examples (i) and (ii) $f \cap g$ while $f$ is not athwart to $g$ in (iii) and (iv).

We observe that, for all $n \geqslant 1$, if $f$ is a convex embedding of the $n$-sphere $S^{n}$ and if $g(N)$ is inside $f\left(S^{n}\right)$ then $f A g$. However convexity of $f$ is obviously not essential for athwartness. It is natural to consider whether any analogue of case (i) in Figure 1 exists for $n>1$. In fact, we shall prove the following two main theorems which give necessary conditions for athwartness and show that there is an interesting difference between the cases $n=1$ (Theorem 4.1) and $n>1$ (Theorem 5.1). Precise definitions are given in Section 2.

(c) 1984 Australian Mathematical Society $0263-6115 / 84 \$ A 2.00+0.00$ 

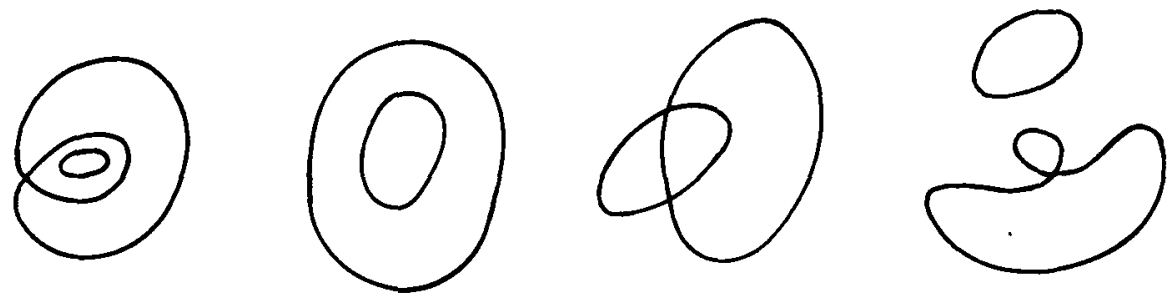

Figure l

THEOREM 4.1. Let $f$ and $g$ be two immersions of $S^{1}$ into $R^{2}$. If $f \mathrm{~A} g$ then the image of one of the immersions is inside all the loops of the other.

THEOREM 5.1. Let $f: M \rightarrow R^{n+1}, g: N \rightarrow R^{n+1}$ be immersions such that $f A g$. Then one of the manifolds, say $M$, is diffeomorphic to $S^{n}, f$ is an embedding with starshaped inside and $g(N)$ is contained in the interior of the kernel of the inside of $f$.

Thanks are due to Dr. L. Lander and Professor H. B. Griffiths for helpful discussions on the case $n=1$. The authors are also grateful to the referee who suggested several improvements and called their attention to [3].

F. J. Craveiro de Carvalho is indebted to INIC-INSTITUTO NACIONAL DE INVESTIGAÇÃ0 CIENTÍFICA-LISBOA-PORTUGAL for financial support.

\section{Notations and definitions}

Throughout the paper we shall be dealing with compact, connected $C^{\infty}$ $n$-manifolds without boundary. All the maps are $C^{\infty}$.

For any immersion $f: M \rightarrow R^{n+1}$ the tangent $n$-plane to $f(M)$ at $f(x)$ will be denoted by $T_{x}$ and is the affine $n$-dimensional subspace of $R^{n+1}$ determined by $f(x)$ and $f_{* x}\left(T_{x} M\right)$, where $f_{* x}: T_{x} M \rightarrow T_{f(x)} R^{n+1} \equiv R^{n+1}$. Such an immersion induces a $C^{\infty}$ map $F: M \rightarrow R_{n}^{n+1}$, where $R_{n}^{n+1}$ denotes the Grassmannian of affine $n$-planes in $R^{n+1}$ [5].

Transversality will be denoted by the usual symbol $\pitchfork$. Thus, from Section 1, if $f: M \rightarrow R^{n+1}$ and $g: N \rightarrow R^{n+1}$ are immersions then $f A g$ if and only if $T_{x} \pitchfork T_{y}$, for any $x \in M, y \in N$.

A loop is a $C^{\infty}$ map $g:[a, b] \rightarrow R^{2}$ such that $g \mid[a, b)$ is injective, $g^{\prime}(t) \neq 0$ for $t \in[a, b]$, and $g(a)=g(b)$. A loop is a Jordan curve and therefore the complement of its image in $R^{2}$ consists of two disjoint open connected subsets of $R^{2}$ 


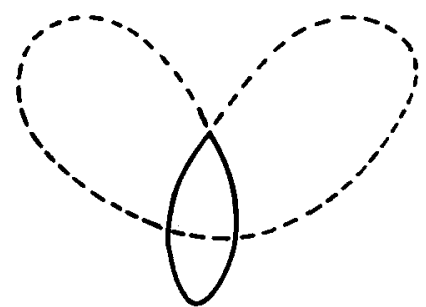

Figure 2

according to the Jordan Curve Theorem [4]. Of these two subsets one is bounded and will be called the inside of the loop $g$ (or the inside of $g([a, b])$ ) while the other is unbounded and is called the outside. If a set $S$ is contained in the inside (outside) of a loop $g$ we say that $S$ is inside (outside) $g$ (or $g([a, b])$ ).

If we look at an immersion of $S^{1}$ into $R^{2}$ as a periodic map $f: R \rightarrow R^{2}$ of period one we can speak of loops of an immersion. We shall not distinguish between two loops of $f$ with the same image in $R^{2}$. Figure 2 illustrates this idea: the image of the "cloverleaf" immersion of $S^{1}$ is indicated by a broken line with the image of one of its six loops shown as a solid line.

If $f: M \rightarrow R^{n+1}$ is an embedding then the complement of $f(M)$ in $R^{n+1}$ is the union of two disjoint open connected sets $B$ and $U$ of which $B$ is bounded and $U$ is unbounded [1]. We say that $B$ is the inside of $f($ or $f(M)$ ) and $U$ is the outside. As before we shall speak of being inside (outside) $f$ (or $f(M)$ ).

If $V$ is a subset of $R^{n+1}$ the kernel of $V$ is the set $\{p \in V \mid t p+(1-t) q \in V$ for all $q \in V$ and $0 \leqslant t \leqslant 1\}$.

\section{A basic result}

TheOREM 3.1. Let $f: M \rightarrow R^{n+1}$ and $g: N \rightarrow R^{n+1}$ be immersions. If $f(M)$ has two tangent n-planes such that one meets $g(N)$ and the other does not then $f$ is not athwart to $g$.

Proof. Assume that, under the above hypothesis, $f A g$. Then $g \pitchfork T_{x}$, for any $x \in M$.

Let $U$ denote the set $\left\{x \in M \mid T_{x} \cap g(N) \neq \varnothing\right\}$. This set and its complement are both non-empty. Since $g(N)$ is compact then $U$ is obviously closed. We show next that it is also open.

Let $x \in U$. By transversality there exist $y_{1}, y_{2}$ in $N$ such that $g\left(y_{1}\right)$ and $g\left(y_{2}\right)$ do not lie in the same half-space complementary to $T_{x}$. The set of hyperplanes 
which separate $g\left(y_{1}\right)$ and $g\left(y_{2}\right)$ is open in $R_{n}^{n+1}$. Therefore, since the map induced by $f$ is continuous, there exists an open neighbourhood $U_{x}$ of $x$ such that, for any $y \in U_{x}, T_{y}$ separates $g\left(y_{1}\right)$ and $g\left(y_{2}\right)$ and so $T_{y} \cap g(N) \neq \varnothing$.

Having proved that $U$ is simultaneously open and closed we conclude that $M$ is not connected. Consequently athwartness must fail somewhere.

COROLlARY 3.1. Let $f: M \rightarrow R^{n+1}$ and $g: N \rightarrow R^{n+1}$ be immersions such that $f(M) \cap g(N) \neq \varnothing$. Then $f$ is not athwart to $g$.

Proof. Suppose that $f=\left(f_{i}\right), g=\left(g_{i}\right), i=1, \ldots, n$. Looking, for instance, at the absolute maxima of $f_{1}$ and $g_{1}$ we see that either $f(M)$ and $g(N)$ have a common tangent $n$-place or one of them has a tangent $n$-plane which does not meet the other. Since $f(M) \cap g(N) \neq \varnothing$ we are then in the position of the previous theorem.

We remark that, for $n=1$, Corollary 3.1 is also a corollary of Theorem 4 in [2]. From now on we shall deal with the cases $n=1, n \geqslant 2$ separately.

\section{Plane curves}

THEOREM 4.1. Let $f$ and $g$ be two immersions of $S^{1}$ into $R^{2}$. If $f \mathrm{~A} g$ then the image of one of the immersions is inside all the loops of the other.

Proof. Suppose that neither of the images lies inside all the loops of the other. Then either $f\left(S^{1}\right) \cap g\left(S^{1}\right) \neq \varnothing$ or $f\left(S^{1}\right) \cap g\left(S^{1}\right)=\varnothing$. If $f\left(S^{1}\right) \cap g\left(S^{1}\right) \neq \varnothing$, by Corollary 3.1, there is a common tangent. If $f\left(S^{1}\right) \cap g\left(S^{1}\right)=\varnothing$ then we must have two loops, one of each curve, such that neither of them is inside the other. As before, we can assume that there is a tangent to one of the images which does not intersect the other and again we are in the position of Theorem 3.1 in view of the following lemma.

LEMMA 4.1. Let $f:[a, b] \rightarrow R^{2}$ be a loop and $p$ an outside point. Then at least one tangent to the loop passes through $p$.

Proof. We shall assume that no tangent line to $f$ passes through $p$. Let $\Psi:[a, b] \rightarrow S^{1}$ be given by $\Psi(t)=(f(t)-p) /\|f(t)-p\|$. Consider the covering map: $g: R \rightarrow S^{1}$, with $g(t)=(\cos 2 \Pi t, \sin 2 \Pi t)$. Let $x \in R$ be such that $g(x)=$ $\Psi(a)=\Psi(b)$ and $\tilde{\Psi}$ the lift of $\Psi$ which starts at $x$. The winding number of $f$ relative to $p$ is given by $\tilde{\Psi}(b)-\tilde{\Psi}(a)$. Since we are assuming that no tangent line 
passes through $p, \Psi$ has no critical points and the same happens to $\tilde{\Psi}$. This map is either increasing or decreasing and thus the winding number is non-zero. Therefore, $p$ is an inside point.

\section{Hypersurfaces}

THEOREM 5.1. Let $f: M \rightarrow R^{n+1}, g: N \rightarrow R^{n+1}$ be immersions such that $f A g$. Then one of the manifolds, say $M$, is diffeomorphic to $S^{n}, f$ is an embedding with starshaped inside and $g(N)$ is contained in the interior of the kernel of the inside of $f$.

Proof. We shall use the following remarkable result due to Halpern [3].

Let $f: M \rightarrow R^{n+1}, \operatorname{dim} M=n$, be an immersion. If $\cup_{x \in M} T_{x} \neq R^{n+1}$ then $M$ is diffeomorphic to $S^{n}, f$ is an embedding, the inside of $f(M)$ is starshaped and $R^{n+1} \backslash \cup_{x \in M} T_{x}$ is the interior of the kernel of the inside of $f$.

Since $f A g$, there exists a tangent $n$-plane to one of the images, say $f(M)$, which does not meet the other, as in Section 3. By Theorem 3.1, no tangent to $f(M)$ meets $g(N)$. The theorem now follows from Halpern's result.

Added in Proof. The conditions of Theorem 5.1 are not only necessary but also sufficient [3].

\section{References}

[1] V. Guillemin and A. Pollack, Differential topology (Prentice Hall, Englewood Cliffs, N. J., 1974).

[2] B. Halpern, 'Global theorems for closed plane curves', Bull. Amer. Math. Soc. 76 (1970), $96-100$.

[3] B. Halpern, 'On the immersion of an $n$-dimensional manifold in $n+1$-dimensional euclidean space', Proc. Amer. Math. Soc. 30 (1971), 181-184.

[4] M. H. A. Newman, Topology of plane sets (Cambridge University Press, 1954).

[5] S. A. Robertson, 'The dual of a height function', J. London Math. Soc. (2) 8 (1974), 187-192.

Faculty of Mathematical Studies

The University

Southampton, S09 5NH

England .
Departamento de Matemática

Universidade de Coimbra

3000 Coimbra

Portugal 